\title{
PREMATURE SHUT OFF POSSIBILITY PREDICTION FOR AUTOMOTIVE FUEL TANK SYSTEM
}

\author{
F. K. Maruyama, \\ K. P. Burr \\ Universidade Federal do ABC \\ Centro de Engenharia, Modelagem e \\ Ciências Sociais Aplicadas - CECS \\ Av. dos Estados, 5001. Bairro Bangu. \\ Santo André - SP - Brasil \\ flavio.koiti@gmail.com \\ karl.burr@ufabc.edu.br \\ Received: Dec 07, 2020 \\ Revised: Dec 27, 2020 \\ Accepted:Fev 02, 2021
}

\section{ABSTRACT}

Prediction of the possibility of a vehicle fuel tank supply process premature shut off is of main concern for the automotive industry. The main objective of this work is to show that premature shut off can be explained by the absence of a steady state after the initial transient in the fuel supply process. The vehicle fuel tank considered in this work is composed only of a rectilinear pipe inclined with respect to the horizontal and connected to the top of a rectangular tank. The counter-current two-phase flow in the pipe is modeled using the one-dimensional drift flux model and the tank is modeled as a control volume where mass conservation for each phase is considered. Linear stability analysis of the two-phase flow model shows the absence of the steady state phase for a range of liquid flow rates before the flooding condition is achieved as a function of tube inclination and length. This fact suggests that premature shut off occurs due to the absence of a steady state regime phase during the fuel supply process.

Keywords: vehicle fuel tank, premature shut off, counter-current two-phase flow, drift-flux model, linear stability analysis.

\section{NOMENCLATURE}

$\mathrm{A}_{\mathrm{p}} \quad$ pipe cross-sectional area, $\mathrm{m}^{2}$

$\mathrm{C}$ Wallis flooding correlation parameter

$\mathrm{C}_{0} \quad$ distribution parameter

$\mathrm{C}_{1} \quad$ integration constant

C submatrix of the Kroneker-Weierstrass Canonical form which has finite eigenvalues

D pipe diameter, $\mathrm{m}$

$f_{g} \quad$ wall friction factor for the gas phase

$f_{i} \quad$ gas-liquid interface friction factor

$f_{1} \quad$ wall friction factor for the liquid phase

$f_{m} \quad$ wall friction factor for the gas-liquid mixture

g gravitational acceleration constant, $\mathrm{m} / \mathrm{s}^{2}$

$\mathrm{h}$ pipe cross section local water depth, $\mathrm{m}$

$\mathrm{H} \quad$ total tank height , $\mathrm{m}$

$\mathbf{H}$ jacobian matrix of vector $\mathbf{h}$

h vector with discretized flow governing equations

identity matrix

superficial velocity

pipe length, $m$

Wallis flooding correlation parameter mass matrix

number of nodes in the pipe length discretization

$\mathrm{N}_{\mathrm{e}} \quad$ number of finite eigenvalues in the KronekerWeierstrass Canonical form
N nilpotent submatrix of the KronekerWeierstrass Canonical form

$\mathrm{P} \quad$ pressure along the pipe

$\mathrm{P}_{\mathrm{atm}} \quad$ atmospheric pressure, $\mathrm{Pa}$

$\mathrm{P}_{\mathrm{b}} \quad$ gas pressure in the tank

$\mathrm{Q}_{10}$ liquid volumetric flow rate at pipe entrance, $\mathrm{m}^{3} / \mathrm{s}$

Q right transformation matrix for the KronekerWeierstrass Canonical form

$\mathrm{Re}_{\mathrm{m}} \quad$ gas-liquid mixture Reynolds number

$\mathrm{Re}_{\mathrm{g}} \quad$ gas phase Reynolds number

$\mathrm{Re}_{1} \quad$ liquid phase Reynolds number

$\mathrm{R}_{\mathrm{g}} \quad$ gas constant, J/kg.K

$\mathbf{R}$ left transformation matrix for the KronekerWeierstrass Canonical form

$\mathrm{s} \quad$ pipe length parameterization

$\mathrm{S} \quad$ phase wetted wall perimeter, $\mathrm{m}$

$\mathrm{t}$ time,

$\mathrm{t}_{0} \quad$ initial instant, $\mathrm{s}$

$\mathrm{T} \quad$ gas temperature, $\mathrm{K}$

$\mathrm{T}_{\mathrm{T}} \quad$ remainning filling tank time estimate

$\mathrm{u} \quad$ weighted mean fluid velocity

$\mathrm{u}_{\mathrm{i}} \quad$ gas-liquid interface velocity

$\mathrm{V}_{\mathrm{gj}} \quad$ void-weighted mean drift velocity, $\mathrm{m} / \mathrm{s}$

$\mathbf{x}$ vector with dependent variables values at pipe length discretization nodes

$\tilde{\boldsymbol{x}} \quad$ vector with stationary state dependent variables values at pipe length discretization nodes 
vector with stationary state perturbation dependent variables values at pipe length discretization nodes

$\overline{\boldsymbol{y}}$ transformation of variables $\overline{\boldsymbol{x}}$

$\overline{\boldsymbol{y}}_{\mathbf{1}}, \overline{\boldsymbol{y}}_{\mathbf{2}} \quad$ split of vector $\overline{\boldsymbol{y}}$ in two parts.

$\left.\bar{y}_{1}\right)_{n},\left(y_{2}\right)_{n} \quad$ n-th component of vectors $\overline{\boldsymbol{y}}_{1}$ and $\overline{\boldsymbol{y}}_{2}$ respectively

\section{Greek symbols}

$\alpha \quad$ void fraction

$\beta_{\mathrm{n}} \quad \mathrm{n}$-th component of integration constant

$\delta$ real part of $\lambda_{\max }$

$\delta_{\mu} \quad$ ratio between the liquid and gas dynamic viscosities

$\epsilon \quad$ the pipe wall roughness, $m$

$\varepsilon \quad$ ratio between the pipe cross sectional area and the tank area

$\gamma \pi$ defines the angle used to define the pipe wall liquid wet perimeter

$\gamma_{\mathrm{i}} \pi$ defines the angle used to define the gas-liquid interface length in terms of pipe diameter.

$\lambda_{\mathrm{n}} \quad \mathrm{n}$-th finite eigenvalue of the matrix pencil (M,H)

$\lambda_{\max } \quad$ finite eigenvalue with largest real part

$\mathrm{H} \quad$ tank water depth, $\mathrm{m}$

$\rho \quad$ fluid density, $\mathrm{kg} / \mathrm{m}^{3}$

$\rho_{\mathrm{m}} \quad$ non-dimensional gas-liquid mixture density

$\pi_{\mathrm{D}}, \pi_{\mathrm{F}}, \pi_{\mathrm{G}}, \pi_{\mathrm{i}} \quad$ non-dimensional numbers

$\mu \quad$ dynamic viscosity, $\mathrm{kg} /(\mathrm{m} . \mathrm{s})$

$\theta$ pipe angle with respect to the horizontal, radians

$v \quad$ nilpotency of matrix $\mathbf{N}$

$\sigma \quad$ air-water interface surface tension, $\mathrm{N} / \mathrm{m}$

$\tau_{\mathrm{m}} \quad$ non-dimensional wall shear stress model for the gas-liquid mixture.

\section{Subscript}

1 variable value at the first pipe discretization node

b pipe end (junction between pipe and tank)

g gas phase

1 liquid phase

$\mathrm{m} \quad$ gas-liquid mixture

n n-th component of a vector

p pipe

T tank

i gás-liquid interface

$\mathrm{N}$ variable value at the $\mathrm{N}$-th pipe discretization node

\section{Superscript}

* non-dimensional variable.

$\simeq \quad$ stationary state dependent variable value

- stationary state perturbation dependent variable value

$\mathrm{T}$ matrix transpose

\section{INTRODUCTION}

The automobile industry considers the problem of a vehicle tank fuel supply process premature shut off of major importance not only to comply with increasingly restrictive environmental and safety standards, but also mainly not to generate customers dissatisfaction.

The vehicle fuel tank system is composed of many parts, where the most commons are the fuel tank, the pipe connecting the pump nozzle to the tank, the auxiliary ventilation duct and a safety valve to avoid fuel leakage in the case of an accident, (see Fackrell et.al. (2003) for an illustration of a vehicle fuel tank system). Figure 1 below illustrate the tank pressure variation for a normal fuel supply process. PHASE I indicates the beginning of the fuel supply process which starts with the opening of the fuel supply pipe, followed by tank pressure equalization with the atmosphere. As the fuel starts filling in the tank, a pressure rise occurs to make the gas inside the tank to flow out to the atmosphere. After this initial transient, the tank pressure stabilizes and a steady state regime is established, which is the PHASE II of the fuel supply process. Finally, PHASE III sets in when the tank pressure rises and a pressure wave reaches the fuel nozzle pressure sensor, which shuts down the fuel supply process.

The fuel supply process can go wrong in different ways, which are described in Godbille et al. (2007) and in Fackrell et al. (2003). They mention the spill back and the premature shut off. The spill back can be divided in the spit off when fuel drops flow away from the pipe entrance and the well back when slugs of fuel flow off the pipe entrance. Both phenomena are result of pipe flooding, when the pipe counter-current twophase flow cannot be sustained for the liquid volumetric flow rate supplied by the fuel pump.

Different from the spill back, the premature shut off does not pose a problem for the fuel supply process operator, but may cause dissatisfaction for the consumer since the tank ends up just partially filled up. The supply process when premature shut off occurs is similar to a normal supply process, however with a pressure rise before the tank is filled up similar to the pressure increase observed in PHASE III of the normal fuel supply process. According to Mastroianni et al. (2011), the premature shut off may be caused by many factors, just as poor pipe geometry design, sharp pipe curvature near the pipe inlet, pipe diameter restrictions and safety valve and auxiliary ventilation pipe geometry.

The premature shut off may happen if PHASE II of the normal fuel supply process does not occur and the transient in PHASE I of the supply process has a pressure rise large enough to cause the fuel pump shutdown. This possibility is studied in this work through the linear stability analysis.

The PHASE II steady state of the fuel supply process is a stable stationary state of an adequate 
mathematical model for the counter-current two-phase flow in the vehicle fuel tank system.

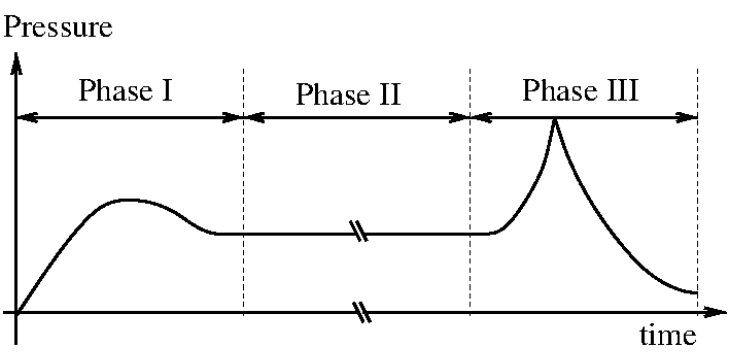

Figure 1. Tank pressure evolution during normal fuel supply process

If the stationary state which represents the PHASE II steady state loses stability, PHASE II of the fuel supply process will not exist, which opens the possibility of a premature shut off. The main objective of this work is to suggest that the premature shut off can be explained by the absence of the steady state regime in the fuel supply process.

The literature about modeling counter-current two-phase flow in vehicle fuel tank is limited. Fackrell et al. (2003) modeled the vehicle fuel tank system parts as a series of four control volumes and four valves. The liquid is assumed incompressible and the process isothermal. Mastroianni et al. (2011) compares experimental results with simulation of Fackrell et al. (2003) model. The simulation results agree well with experiments of normal fuel supply process. It also gives reasonable prediction for premature shut off, but not for all experimental situations where premature shut off happens.

Many authors made use of computational fluid dynamics to simulate the two-phase flow in the vehicle fuel tank system. Examples of this research line are Sinha et al. (1998), Banerjee et al. (2001), Banerjee et al. (2002). Experimental results in the literature are also limited. Mastroianni et al. (2011) studied experimentally the influence of the diameter of the auxiliary duct, of the fuel vaporization rate and of the fuel flow rate in the occurrence of premature shut off.

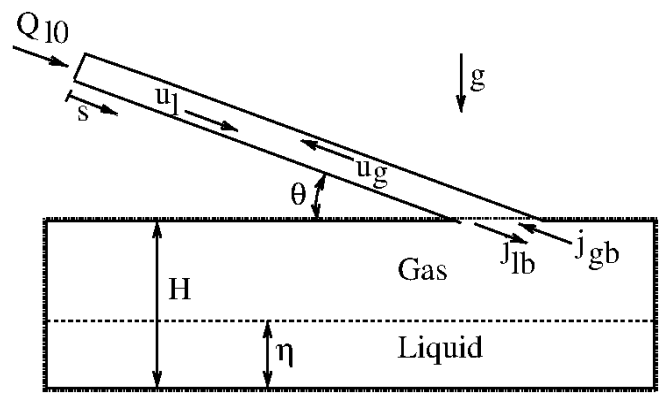

Figure 2. Pipe-tank system geometry and tank control volume
The vehicle fuel tank system considered in this work is composed of a straight pipe inclined with an angle $\theta$ with respect to the horizontal and connected to the top of a tank with uniform rectangular horizontal cross section, as illustrated in Fig. 2 above. A one-dimensional flow model is used to represent the counter-current two-phase flow in the pipe and the fuel tank is modeled as a control volume.

In the next section, details of the one-dimensional model for the pipe counter-current two-phase flow are given and the tank flow model is discussed. The linear stability analysis of the vehicle fuel tank system considered in this work is discussed in the third section. Results in the form of stability maps are given in the fourth section and a discussion and conclusions are presented in the fifth section.

\section{FUEL TANK SYSTEM TWO-PHASE FLOW MODEL}

As mentioned above, the vehicle fuel system is composed by a rectangular tank and a rectilinear pipe inclined with an angle $\theta$ with respect to the horizontal. The pipe is connected to the top of the tank as illustrated in the figure Fig. 2. The counter-current two-phase flow in the pipe is modeled by an one-dimensional drift-flux model, where mass conservation for each phase and a linear momentum equation for the mixture are considered plus a closure relation, which is a kinematic relation that fixes the relative velocity between phases and is pattern dependent. A distinction only between stratified and non-stratified patterns is considered. The linear momentum equation is also pattern dependent. The tank is modeled as a control volume where mass conservation for each phase is considered. The gas is assumed to behave as a perfect gas. The fuel supply process is assumed isothermal in both pipe and tank. The liquid in the tank is assumed in hydrostatic equilibrium and the gas pressure in the tank is assumed uniform.

The two-phase flow governing equations in the pipe and tank are given in non-dimensional form. The variable $\mathbb{Q}_{10}$ are made non-dAmensional by

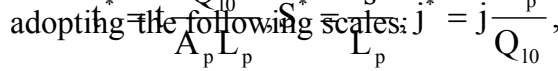

$$
u^{*}=u \frac{A_{p}}{Q_{10}}, P^{*}=\frac{P}{\rho_{1} R_{g} T}
$$

where $Q_{10}$ is the liquid volumetric flow rate that enters the pipe inlet, $A_{p}$ is the pipe cross-sectional area, $L_{p}$ is the pipe length, $\rho_{1}$ is the liquid density, $R_{g}$ is the gas constant, $\mathrm{T}$ is the gas temperature, $\mathrm{P}$ is the pressure along the pipe, $\mathrm{j}$ stands for superficial 
velocity, $\mathrm{u}$ stands for fluid velocity, $\mathrm{s}$ is the pipe length parameterization and $t$ represents time.

The next subsection describes in detail the onedimensional drift-flux model adopted, the form of the linear momentum equation, the kinematic relation for the stratified and non-stratified flow patterns and a transition criteria between flow patterns. The governing equation for the flow in the tank is discussed in the subsection following the next subsection.

\section{Pipe Two-Phase Flow Model}

To simplify the notation the super script $*$ is omitted in what follows. All variables are nondimensional. For the one-dimensional drift flux model for the counter-current two-phase flow in the pipe, mass conservation for each phase is considered. Liquid mass conservation is given by the equation

$$
-\frac{\partial \alpha}{\partial t}+\frac{\partial j_{1}}{\partial s}=0
$$

where $\alpha$ is the void fraction, $\mathrm{j}_{1}$ is the liquid superficial velocity. Gas mass conservation is given by the equation

$$
\frac{\partial}{\partial t}(P \alpha)+\frac{\partial}{\partial s}\left(P j_{g}\right)=0
$$

where $\mathrm{j}_{\mathrm{g}}$ is the gas superficial velocity.

As mentioned before, the mixture momentum equation is pattern dependent and obtained by adding the one-dimensional two-fluid model momentum equation for each phase. At the pipe cross section the pressure is assumed uniform and the friction force is modeled according to the flow pattern. When the flow is stratified the friction force is basically the sum of the liquid wall friction and the gas wall friction. When the flow pattern is non-stratified, the friction force is modeled as the wall friction of a pseudo-fluid (gasliquid mixture). The linear momentum equation has the form

$$
\begin{aligned}
& \pi_{\mathrm{I}}\left\{\frac{\partial \mathrm{j}_{1}}{\partial \mathrm{t}}+\frac{\partial}{\partial \mathrm{t}}\left(\mathrm{Pj}_{\mathrm{g}}\right)+\frac{\partial}{\partial \mathrm{s}}\left(\mathrm{u}_{1} \mathrm{j}_{1}\right)+\frac{\partial}{\partial \mathrm{s}}\left(\mathrm{Pu}_{\mathrm{g}} \mathrm{j}_{\mathrm{g}}\right)\right\} \\
& +\frac{\partial \mathrm{P}}{\partial \mathrm{s}}-\rho_{\mathrm{m}} \pi_{\mathrm{G}} \sin \theta+2 \pi_{\mathrm{F}} \tau_{\mathrm{m}}=0
\end{aligned}
$$

where $\mathrm{u}_{1}$ is the weighted mean liquid velocity, $\mathrm{u}_{\mathrm{g}}$ is the weighted mean gas velocity, and the nondimensional number $\pi_{\mathrm{I}}, \pi_{\mathrm{G}}$ and $\pi_{\mathrm{F}}$ are nondimensional numbers given by the equations

$$
\begin{gathered}
\pi_{\mathrm{I}}=\mathrm{Q}_{10}^{2} /\left(\mathrm{R}_{\mathrm{g}} \mathrm{TA}_{\mathrm{p}}^{2}\right) \\
\pi_{\mathrm{G}}=\mathrm{gL}_{\mathrm{p}} /\left(\mathrm{R}_{\mathrm{g}} \mathrm{T}\right) \\
\pi_{\mathrm{F}}=\frac{\mathrm{L}_{\mathrm{p}}}{\mathrm{D}} \pi_{\mathrm{I}}
\end{gathered}
$$

where $\mathrm{D}$ is the pipe diameter. The mixture density $\rho_{\mathrm{m}}$ is given by the equation

$$
\rho_{\mathrm{m}}=(1-\alpha+\mathrm{P} \alpha)
$$

and $\tau_{m}$ is the non-dimensional wall shear stress for the liquid and gas mixture, which is flow pattern dependent. For non-stratified flow $\tau_{m}$ is given by:

- For turbulent flow $\left(\operatorname{Re}_{m}>2200\right)$

$$
\tau_{\mathrm{m}}=\rho_{\mathrm{m}} \mathrm{f}_{\mathrm{m}}\left(\operatorname{Re}_{\mathrm{m}}, \in / \mathrm{D}\right) \mathrm{j}|\mathrm{j}|
$$

where $\mathrm{j}$ is the total superficial velocity, $\varepsilon$ is the pipe wall roughness and $f_{m}\left(\operatorname{Re}_{m}, \in / D\right)$ is the Fanning friction factor. $\mathrm{Re}_{\mathrm{m}}$ is the gas-liquid mixture Reynolds number given by the equation

$$
\operatorname{Re}_{m=} \frac{\rho_{1} \mathrm{DQ}_{10}}{\mathrm{~A}_{\mathrm{p}} \mu_{\prime_{1}}} \frac{1-\alpha+\mathrm{P} \alpha}{1-\alpha+\delta_{\mu} \alpha}
$$

where $\rho_{1}$ is the liquid density, $\mu_{1}$ is the liquid dynamic viscosity and $\delta_{\mu}=\mu_{\mathrm{g}} / \mu_{1}$, where $\mu_{\mathrm{g}}$ is the gas dynamic viscosity.

- For laminar flow $\left(\operatorname{Re}_{\mathrm{m}}<2000\right)$

$$
\tau_{\mathrm{m}}=\frac{16}{\operatorname{Re}_{1}} \mathrm{j}\left[(1-\alpha)+\delta_{\mu} \alpha\right]
$$

with

$$
\operatorname{Re}_{1}=\frac{\rho_{1} \mathrm{D}}{\mu_{\prime_{1}}} \frac{\mathrm{Q}_{10}}{\mathrm{~A}}
$$

- For transition between laminar and turbulent flow $\left(2000 \leq \operatorname{Re}_{m} \leq 2200\right), \tau_{m}$ is given by Eq. (7), but with the Fanning friction factor as a linear interpolation between its laminar $\left(\operatorname{Re}_{m}=2000\right) \quad$ and turbulent form $\left(\operatorname{Re}_{m}=2200\right)$

For stratified flow $\tau_{m}$ is given by 


$$
\begin{aligned}
& \tau_{\mathrm{m}}=\mathrm{f}_{1}\left(\operatorname{Re}_{1}, \in / D\right) \mathrm{j}_{1}\left|\mathrm{j}_{1}\right| \frac{\gamma}{(1-\alpha)^{2}} \\
& +\operatorname{Pf}_{\mathrm{g}}\left(\operatorname{Re}_{\mathrm{g}}, \in / D\right) \mathrm{j}_{\mathrm{g}}\left|\mathrm{j}_{\mathrm{g}}\right| \frac{(1-\gamma)}{\alpha^{2}}
\end{aligned}
$$

where $f_{1}$ and $f_{g}$ are, respectively, the liquid and gas phases Fanning friction factors, $\mathrm{Re}_{l}$ and $\mathrm{Re}_{g}$ are respectively the liquid and gas Reynolds numbers given by the equations

$$
\begin{gathered}
\operatorname{Re}_{1=} \frac{\rho_{1} Q_{10} D}{\mu_{1} A_{p}}\left|j_{1}\right| \\
\operatorname{Re}_{g=} \frac{\rho_{g} Q_{10} D}{\mu_{g} A_{p}} \frac{\left|j_{g}\right|}{1-\gamma-\gamma_{i}}
\end{gathered}
$$

where $\gamma$ is an implicit function of the void fraction $\alpha$ given by the equation

$$
\alpha=1-\gamma+\frac{1}{\pi} \sin (\pi \gamma)
$$

and $\gamma_{i}$ is given in terms of $\gamma$ according to the equation

$$
\gamma_{\mathrm{i}}=\frac{1}{\pi} \sin (\pi \gamma)
$$

An explicit equation for the Fanning friction factors $f_{m}, f_{1}$ and $f_{g}$ are given in Chen (1979).

Notice that the modeling of the stratified flow assumes a flat interface between gas and liquid. This is implicit in the modeling of the gas, liquid and gasliquid interface perimeters, given in terms of the parameters $\gamma$ and $\gamma_{\mathrm{i}}$ respectively as $\mathrm{S}_{1}=\gamma \pi \mathrm{D}$, $\mathrm{S}_{\mathrm{g}}=(1-\gamma) \pi \mathrm{D}$ and $\mathrm{S}_{\mathrm{i}}=\gamma_{\mathrm{i}} \pi \mathrm{D}$. This assumption does not holds for large pipe inclinations since the gravitational effects in the transverse flow direction becomes weak and the surface tension effects dominate, causing the interface to deform.

To close the model a flow pattern dependent kinematic relation fixing the relative velocity between phases is expressed as

$$
\mathrm{A}\left(\mathrm{j}_{1}, \mathrm{j}_{\mathrm{g}}, \mathrm{P}, \alpha\right)=0
$$

The appropriate functional form for the kinematic relation for stratified (non-stratified) flow pattern is given in the next paragraph. To be able to use the right form of the kinematic relation a transition criterion between stratified and non-stratified flow pattern is needed. Such criterion for counter-current stratified flow is given after the kinematic relations for stratified and non-stratified flow pattern are presented. In addition, the relation between the liquid (gas) superficial velocity and the liquid (gas) weighted mean velocity are necessary. These relations are given by the equations

$$
(1-\alpha) \mathrm{u}_{1}-\mathrm{j}_{1}=0
$$

And

$$
\alpha u_{g}-j_{g}=0
$$

The kinematic relation for stratified flow is obtained from the one-dimensional two-fluid model by subtracting the gas linear momentum equation from the liquid linear momentum equation under the hypothesis of fully developed flow regime. Under this assumption, the resulting equation is the balance among the gas and liquid wall friction force, the airliquid interface friction force and the gravitational force component in the tube axis direction, and is given by the equation

$$
\begin{aligned}
& A\left(j_{1}, j_{g}, P, \alpha\right)=P f_{i}\left(\frac{j_{g}}{\alpha}+u_{i}\right)\left|\frac{j_{g}}{\alpha}-u_{i}\right| \frac{\gamma_{i}}{\alpha(1-\alpha)} \\
& -f_{1} \frac{j_{1}\left|j_{1}\right|}{(1-\alpha)^{3}} \gamma+P f_{g} j_{g}\left|j_{g}\right| \frac{(1-\gamma)}{\alpha^{3}}+\frac{1}{2} \pi_{D}(1-P) \sin \theta
\end{aligned}
$$

where $f_{i}$ is the interface friction factor, $u_{i}$ is the interface velocity, $f_{1}$ and $f_{g}$ are the wall friction factors for the liquid and gas, respectively. They are functions of the non-dimensional wall roughness $\varepsilon / \mathrm{D}$ and the respective Reynolds numbers $\mathrm{Re}_{l}$ and $\mathrm{Re}_{\mathrm{g}}$ given, respectively, by Eqs. (12) and (13). $\pi_{\mathrm{D}}=\mathrm{gDQ}_{10}^{2} / \mathrm{A}_{\mathrm{p}}^{2}$ is a non-dimensional number.

The interfacial friction factor $f_{i}$ is assumed constant and equal 0.0142 . This is the interfacial friction factor for co-current wavy stratified flow with small amplitude waves given by Eq. (5.67) of Shoham (2005).

When the flow pattern is not stratified the onedimensional drift flux relation is used as a kinematic constitutive relation to close the system of equations and is given by the equation

$$
A\left(j_{1}, j_{g}, P, \alpha\right)=j_{g}-\alpha\left(C_{0} j+V_{g j}\right)
$$

where the distribution parameter $\mathrm{C}_{0}$ and the voidweighted mean drift velocity $\mathrm{V}_{\mathrm{gj}}$ are given by the 
Chexal-Lellouche correlation, which is independent of the flow pattern, applicable to a full range of thermodynamic conditions and geometries, and covers air-water counter-current flows. The ChexalLellouche correlation can be found in Hibiki (2019), Chexal et al. (1997) and Chexal et al. (1992)

The values given by the Chexal-Lellouch correlation for the mean drift velocity $\mathrm{V}_{\mathrm{gj}}$ for countercurrent flow lead to counter-current flooding limit (CCFL) line well below the flooding line obtained from experimental results for air-water flow in inclined pipes reported in the literature (see Ghiaasiaan et al. (1995)). This behavior was already mentioned by Takeuchi et al. (1992), where the authors compare the drift velocity predicted by the Chexal-Lellouche correlation with the drift velocity predicted by others correlations and showed that the Chexal-Lellouch correlations gives smaller values for the drift velocity.

For the drift flux relation to give the CCFL line which matches the experimental flooding line given in Ghiaasiaan et al. (1995) for inclined tubes and given in terms of Wallis flooding correlation

$$
\left(\mathrm{j}_{\mathrm{g}}^{*}\right)+\mathrm{m}\left(\mathrm{j}_{1}^{*}\right)^{1 / 2}=\mathrm{C}
$$

where

$$
j_{k}^{*}=\frac{Q_{10}}{A} j_{k}\left[\frac{\rho_{k}}{g D\left(\rho_{1}-\rho_{g}\right)}\right]^{1 / 2}
$$

with $\mathrm{k}=\mathrm{g}$ or $\mathrm{l}, \mathrm{m}=0.66$ and $\mathrm{C}=0.6$, the mean drift velocity $\mathrm{V}_{\mathrm{gj}}$ is now given as a function of the distribution parameter $C_{0}$ given by the ChexalLellouche correlation, according to the equation

$$
V_{g j}=C^{2}\left[\frac{g D\left(\rho_{1}-\rho_{g}\right)}{C}\right]^{1 / 2} \frac{C_{0}}{\frac{C_{0} \alpha}{C_{0} \alpha-1}-m^{2}\left(\frac{\rho_{1}}{\rho_{g}}\right)^{1 / 2}}
$$

Note that this expression gives a dimensional $\mathrm{V}_{\mathrm{g} j}$ To make it non-dimensional just divide it by the adopted velocity scale $Q_{10} / A_{p}$. Equation (22) was obtained using the theory presented in Ohkawa and Lahey $\operatorname{Jr}$ (1980), based in the idea that the CCFL condition occurs at a point of kinematic "choking", when the void fraction perturbation waves no longer propagate. This theory obtains the mean drift velocity $\mathrm{V}_{\mathrm{gj}}$ as a function of the drift parameter $\mathrm{C}_{0}$ such that the CCFL line coincides with the flooding line given by an appropriate flooding correlation.
In summary, for non-stratified flow, the onedimensional drift-flux relation, given by Eq. (20), is used as the kinematic relation to close the system of equations, but with the distribution parameter $C_{0}$ given by the Chexal-Lellouche correlation and the mean drift velocity given by the Eq. (22).

A single transition criterion is used since no distinction is made between stratified smooth and stratified wavy flow. The flow pattern transition criterion is for the transition from the stratified wavy flow to other flow patterns. It is based on the balance between the suction force due to the pressure decrease above the wave crest and the gravitational force component in the direction orthogonal to the tube axis. If the suction force is larger than the gravitational force, the stratified flow pattern is unstable and a nonstratified flow pattern emerges. According to experimental results in counter-current flow in inclined tubes, the waves propagate downstream against the gas flow (see Deendarlianto and Fukano (2005)).

The maximum wave velocity is given by $\sqrt{\mathrm{gh}}$, where $\mathrm{h}$ is the local water depth. Therefore, the wave propagating downstream with the maximum wave velocity with respect to the liquid is the wave most susceptible to grow due to the suction force above its crest. This leads to the following stability criterion: If the inequality given by the equation

$$
\frac{\mid \mathrm{j}_{\mathrm{g}}\left[\left[\mathrm{j}_{\mathrm{g}} \mid(1-\alpha)+\alpha \mathrm{j}_{1}+\mathrm{h}^{*} \alpha \frac{(1-\alpha)}{\mathrm{F}_{1}}\right]\right.}{\alpha^{3}(1-\alpha)} \frac{\mathrm{S}_{\mathrm{i}}{ }^{*}}{1-\mathrm{h}^{*}} \mathrm{~F}^{2}<1
$$

with $\mathrm{h}^{*}=\mathrm{h} / \mathrm{D}, \mathrm{S}_{\mathrm{i}}^{*}=\gamma_{\mathrm{i}} \pi \mathrm{D}$

$$
F^{2}=\left(\frac{Q_{10}}{A_{p}}\right)^{2}\left[\frac{\rho_{g} D}{\left(\rho_{1}-\rho_{g}\right) A_{p} g \cos \theta}\right]
$$

and

$$
F_{1}=\frac{Q_{10}}{A_{p}}\left[\frac{1}{\sqrt{g D \cos \theta}}\right]
$$

is satisfied, the stratified flow is stable. $S_{i}$ is the gasliquid interface perimeter.

\section{Tank Two-Phase Flow Model}

The tank is modeled as a control volume. Mass conservation for both phases is considered. Liquid and gas mass conservation for the control volume illustrated in Fig. 2 leads to two coupled differential equations. The liquid mass governing equation can be integrated in time to give 


$$
\frac{\eta(\mathrm{t})}{\mathrm{L}_{\mathrm{p}}}=\frac{\eta\left(\mathrm{t}_{0}\right)}{\mathrm{L}_{\mathrm{p}}}+\frac{\mathrm{A}_{\mathrm{p}}}{\mathrm{A}_{\mathrm{T}}} \int_{\mathrm{t}_{0}}^{\mathrm{t}} \mathrm{j}_{\mathrm{lb}} \mathrm{dt}
$$

where $\eta$ is the water depth in the tank, $A_{T}$ is the tank cross section in the horizontal plane and $j_{l b}$ is the liquid superficial velocity at the tank inlet. Then, $\eta(t)$ and $\partial \eta / \partial t$ can be eliminated from the gas mass conservation equation, which assumes the form

$$
\left(\frac{H-\eta\left(t_{0}\right)}{L_{p}}-\frac{A_{p}}{A_{T}} \int_{t_{0}}^{t} j_{l b} d t\right) \frac{\partial P_{b}}{\partial t}-P_{b} \frac{A_{p}}{A_{T}}\left(j_{l b}-j_{g b}\right)=0
$$

where $\mathrm{j}_{\mathrm{gb}}$ is the gas superficial velocity at the tank entrance, $\mathrm{P}_{\mathrm{b}}$ is the pressure of the gas inside the tank and $\mathrm{H}$ is the total tank height. Eq. (27) will be used as the tank governing equation.

\section{Compatibility Condition Between Tube and Tank}

The compatibility conditions are the continuity of pressure and gas and liquid superficial velocities at the tube end and tank entrance. They are given by the equations

$$
\begin{gathered}
\mathrm{j}_{1}(\mathrm{~s}=1, \mathrm{t})=\mathrm{j}_{\mathrm{lb}} \\
\mathrm{j}_{\mathrm{g}}(\mathrm{s}=1, \mathrm{t})=\mathrm{j}_{\mathrm{gb}} \\
\mathrm{P}(\mathrm{s}=1, \mathrm{t})=\mathrm{P}_{\mathrm{b}}
\end{gathered}
$$

\section{Boundary Conditions}

The boundary conditions are the atmospheric pressure $P_{a t m}$ and the liquid superficial velocity at tube entrance. They are given by the equations

$$
\begin{gathered}
\mathrm{j}_{1}(\mathrm{~s}=0, \mathrm{t})=1 \\
\mathrm{P}(\mathrm{s}=0, \mathrm{t})=\frac{\mathrm{P}_{\mathrm{atm}}}{\rho_{1} \mathrm{R}_{\mathrm{g}} \mathrm{T}}
\end{gathered}
$$

\section{Space Discretization and Equations in Matrix From}

The flow governing equation for the tank is a differential equation, but the governing equations for the counter-current two-phase flow in the pipe are partial differential equations. To address the linear stability analysis of the tank and pipe flow governing equations in the same form, the pipe flow governing equations are discretized.

The pipe length is discretized in $N-1$ subintervals (a mesh with $N$ nodes). Each conservation equation is integrated in each sub-interval, resulting in a system of $3 N-3$ differential equations. The algebraic relations are imposed at each node, resulting in $3 N$ equations. Finally the two boundary conditions are imposed in the first node and the tank governing equation is written in terms of the variables at the last node using the pipe-tank compatibility relations (Eqs. (28)-(30)). As a result, a system of $6 N$ equations is ob aine $\mathrm{d}$ a $\mathrm{n}$ dep rese in thed a trix for as

$$
\mathbf{M}(\mathbf{x}, t) \frac{\partial}{\partial t} \mathbf{x}=\mathbf{h}(\mathbf{x})
$$

where:

$$
\begin{aligned}
& x^{T}=\left\{j_{1,1} \ldots j_{1, N}, j_{g, 1} \ldots j_{g, N}, P_{1} \ldots P_{N}, \alpha_{1} \ldots \alpha_{N}\right. \text {, } \\
& \left.u_{l, 1} \ldots u_{l, N}, u_{g, 1} \ldots u_{g, N}\right\}
\end{aligned}
$$

\section{LINEAR STABILITY ANALYSIS}

Here the linear stability analysis process of the model for the counter-current two-phase flow in the pipe-tank system considered is described. The first step is to identify the stationary states. Once they are identified, their linear stability is studied. The dependent variables are written as their stationary state value plus a perturbation and then substituted into the counter-current two-phase flow governing equations.

The resulting equations are then subtracted from the governing equations for the stationary states. What is left are the governing equations for the stationary state perturbations, which are nonlinear equations. To simplify these equations, the perturbations are assumed infinitesimal, and under such assumption the nonlinear terms with respect to the perturbations in the perturbations governing equations are eliminated. The resulting linear perturbations governing equations are used to study the stationary states stability. If their solution grows with time, the stationary state is unstable, but if their solution decays with time, the stationary state is stable.

In the next subsection, the stationary states are identified and the process to obtain them is discussed. In the second subsection, the stationary sate perturbation linear governing equations are obtained. Their solution and the resulting stationary state linear stability criterion are given in the third subsection.

\section{Stationary State}

The governing equations for the stationary states are obtained from the governing equations for the 
counter-current two-phase flow in the pipe-tank system by dropping of the derivatives with respect to time. Since the one-dimensional drift-flux model is used to model the counter-current two-phase flow in the pipe, there are two possible void fraction values for counter-current flow, which implies in two stationary states. Since the governing equations for the stationary state are easily obtained from Eqs. (2) - (18) and Eq. (27) by setting the time derivatives to zero, they are not given below, just the process to reduce them to a single equation for the pressure and its boundary conditions is described.

From Eqs. (2) and (31), at the stationary state $\mathrm{j}_{1}(1)=1,0<\mathrm{s}<1$. From Eq. (3) it follows that $P j_{g}=C$ (constant). Then, $j_{g}=C_{1} / P$. From Eq. (27) and the fact that $\mathrm{j}_{\mathrm{lb}}=\mathrm{j}_{1}(\mathrm{~s}=1)=1$, it follows that $\mathrm{j}_{\mathrm{gb}}=-1=\mathrm{j}_{\mathrm{g}}(\mathrm{s}=1)$. From Eqs. (17)-(18), it is possible to write $\partial \mathrm{u}_{1} / \partial \mathrm{s}, \partial \mathrm{u}_{\mathrm{g}} / \partial \mathrm{s}$ and $\partial \alpha / \partial \mathrm{s}$ in terms of $\partial P / \partial s$. Finally, Eq. (4) is used to obtain a differential equation for the pressure, which is given by

$$
\begin{gathered}
\pi_{\mathrm{i}}\left\{-\frac{\mathrm{j}_{1}^{2}}{(\alpha-1)^{2}}\left(\frac{\partial \mathrm{A}}{\partial \alpha}\right)^{-1}\left(\frac{\partial \mathrm{A}}{\partial \mathrm{P}}-\frac{\mathrm{j}_{\mathrm{g}}}{\mathrm{P}} \frac{\partial \mathrm{A}}{\partial \mathrm{j}_{\mathrm{g}}}\right)+\right. \\
\left.\frac{\mathrm{Pj}_{\mathrm{g}}}{\alpha}\left[-\frac{\mathrm{j}_{\mathrm{g}}}{\mathrm{P}}+\mathrm{u}_{\mathrm{g}}\left(\frac{\partial \mathrm{A}}{\partial \alpha}\right)^{-1}\left(\frac{\partial \mathrm{A}}{\partial \mathrm{P}}-\frac{\mathrm{j}_{\mathrm{g}}}{\mathrm{P}} \frac{\partial \mathrm{A}}{\partial \mathrm{j}_{\mathrm{g}}}\right)\right]\right\} \frac{\partial \mathrm{P}}{\partial \mathrm{s}} \\
+\frac{\partial \mathrm{P}}{\partial \mathrm{s}}=\rho_{\mathrm{m}} \pi_{\mathrm{G}} \sin \theta-2 \pi_{\mathrm{F}} \tau_{\mathrm{m}}
\end{gathered}
$$

for $0<s<1$.

This differential equation is part of a boundary value problem since it has boundary conditions at $s=0$ (Eq. (32)) and at $s=1\left(j_{g}=1\right)$. The strategy to solve this boundary value problem is to use the shooting method. A value for $j_{g}$ at $s=0$ is assumed and Eq. (34) is integrated in s from $s=0$ up to $s=1$ and then the boundary condition $j_{g}=-1$ is checked. If this boundary condition is not satisfied, the value of $j_{g}$ at $s=0$ is changed until the boundary condition $j_{g}=-1$ is satisfied. The bisection method is used to perform the search of the right value of $\mathrm{j}_{\mathrm{g}}$ at $\mathrm{s}=0$ to satisfy the boundary condition $j_{g}=-1$ at $s=1$.

\section{Perturbations Governing Equations}

To study the linear stability of the stationary sates identified in the previous section, the dependent variables $\mathbf{x}$ are written as their stationary state value $\widetilde{\mathbf{x}}$ plus a perturbation $\overline{\mathbf{x}}$ and substituted into the counter-current two-phase flow governing equations given in matrix form by Eq. (33), which results in the matrix equation

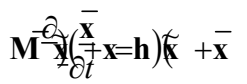

since $\partial \widetilde{\boldsymbol{x}} / \partial t=0$. The perturbations $\overline{\mathbf{x}}$ are assumed infinitesimal $(|\overline{\mathbf{x}}|<<\mathbf{1})$, and then Eq. (35) above is linearized, which gives

$$
\mathbf{M}(\widetilde{\mathbf{x}}) \frac{\partial \overline{\mathbf{x}}}{\partial t}=\mathbf{H}(\widetilde{\mathbf{x}}) \overline{\mathbf{x}}+\mathrm{O}\left(|\overline{\mathbf{x}}|^{2}\right)
$$

where matrix $\mathbf{H}(\widetilde{\boldsymbol{x}})=\frac{\partial \mathbf{h}}{\partial \boldsymbol{x}}(\widetilde{\mathbf{x}})$

\section{Solution and Stability Criterion}

The DAE (differential algebraic equation) obtained in the previous section is non-autonomous since its last equation has a time dependent coefficient. It is the linearized form of the tank flow governing equation given in terms of the perturbation and stationary state variables by the equation

$$
\left(\frac{H}{L p}-\frac{\eta}{L_{p}}-\frac{A_{p}}{A_{T}} \int_{t_{0}}^{t} \widetilde{j}_{1, N} d t\right) \frac{\partial \bar{P}_{N}}{\partial t}-\frac{A_{p}}{A_{T}} \widetilde{P}_{N}\left(\bar{j}_{1, N}-\bar{j}_{g, N}\right)=0
$$

If it is assumed that $A_{p} / A_{T}=\varepsilon<<1$, the tank governing equation above implies that $\partial \overline{\mathrm{P}} \mathrm{N} / \partial \mathrm{t}=\mathrm{O}(\varepsilon)$, very small. The time to fill in the tank is given implicitly by the Eq. (26). If $\eta\left(t_{0}\right)$ is assumed to be the liquid depth in the tank at the beginning of PHASE II of the fuel supply process, where there is a steady state regime (one of the two possibles stationary states is stable), and substituting $\mathrm{j}_{1, \mathrm{~N}}$ for its stable stationary state value $\widetilde{\mathrm{j}}_{\mathrm{l}, \mathrm{N}}$, an estimate for the period of the remaining fuel supply process is

$$
\mathrm{T}_{\mathrm{T}}=\frac{1}{\varepsilon} \frac{\mathrm{H}-\eta\left(\mathrm{t}_{0}\right)}{\mathrm{L}_{\mathrm{p}} \widetilde{\mathrm{j}}_{\mathrm{lb}}}
$$

which is of order $\mathrm{O}(1 / \varepsilon)$. Regarding the stability analysis of the stationary states, there is no need to solve the perturbation governing equations up to time scales of $\mathrm{O}(1 / \varepsilon)$. It is enough to find solutions valid to time scale of $\mathrm{O}(1)$. In this time range, the tank perturbation pressure is at most of $\mathrm{O}(\varepsilon)$, very small. Therefore, up to time scales of $\mathrm{O}(1)$, the tank pressure perturbation can be assumed constant and equal to 
zero. With this assumption, the last line in the matrix equation governing the stationary state perturbations is replaced by

$$
\overline{\mathrm{P}}_{\mathrm{N}}=0
$$

and the coefficients in matrix $\mathbf{M}(\widetilde{\mathbf{x}})$ which multiplies $\partial \overline{\mathrm{P}} \mathrm{N} / \partial \mathrm{t}$ are set to zero. Next, the solution of the resulting system of perturbation equations is addressed. Admit that there exist matrices $\mathbf{R}$ and $\mathbf{Q}$ such that

$$
\mathbf{R M}(\widetilde{\mathbf{x}}) \mathbf{Q}=\left[\begin{array}{ll}
\mathbf{I} & \mathbf{0} \\
\mathbf{0} & \mathbf{N}
\end{array}\right]
$$

and

$$
\mathbf{R H}(\widetilde{\mathbf{x}}) \mathbf{Q}=\left[\begin{array}{ll}
\mathbf{C} & \mathbf{0} \\
\mathbf{0} & \mathbf{I}
\end{array}\right]
$$

where $\boldsymbol{I}$ is the identity matrix of dimension $6 N-N_{e} x 6 N-N_{e}$ in Eq. (41), $C$ is a matrix of dimension $N_{e} x N_{e}$ and its main diagonal contains the $N_{e}$ finite eigenvalues $\lambda_{n}$ of the matrix pencil $(\mathbf{M}, \mathbf{H})$ . Matrix $N$ has dimensions $6 \mathrm{~N}-\mathrm{N}_{\mathrm{e}} \times 6 \mathrm{~N}-\mathrm{N}_{\mathrm{e}}$ and has the property $\mathbf{N}^{k} \neq 0$ for $\mathbf{k}=\mathbf{1}, \ldots, v-\mathbf{1}$, but $\mathbf{N}^{v}=0$ for an integer $v$. As a consequence, the matrix pencil $(\mathbf{M}, \mathbf{H})$ has index $v-1$. It is assumed that this matrix pencil is regular and it has the Kroneker-Weierstrass Canonical form illustrated by the right side of the matrix identities in Eq. (41) and Eq. (42). Now, write $\overline{\boldsymbol{x}}=\boldsymbol{Q} \bar{y}$ and substitute into Eq. (36). Then, the resulting equation is left multiplied by $\mathbf{R}$ and by taking into account Eq. (41) and Eq. (42), the result is

$$
\left[\begin{array}{ll}
\boldsymbol{I} & \mathbf{0} \\
\mathbf{0} & \boldsymbol{N}
\end{array}\right] \frac{d}{d t}\left\{\begin{array}{l}
\overline{\boldsymbol{y}}_{1} \\
\overline{\bar{y}}_{2}
\end{array}\right\}=\left[\begin{array}{ll}
\boldsymbol{C} & \mathbf{0} \\
\mathbf{0} & \boldsymbol{I}
\end{array}\right]\left\{\begin{array}{l}
\overline{\boldsymbol{y}}_{1} \\
\overline{\overline{\boldsymbol{y}}}_{2}
\end{array}\right\}
$$

which has solution $\left(\overline{\mathrm{y}}_{1}\right)_{\mathrm{n}}=\beta_{n} e^{\left(\lambda_{n} t\right)}, n=1, \ldots, N_{e}$, and $\left(\overline{\boldsymbol{y}}_{2}\right)_{\mathrm{n}}=0, n=1, \ldots, 6 N-N_{e} \cdot \lambda_{n}$ are the finite eigenvalues of the matrix pencil $(\mathbf{M}, \mathbf{H})$. Therefore, if all eigenvalues $(\mathbf{M}, \mathbf{H})$ has negative real part, the solution of Eq. (41) decays with time and then the stationary state $\widetilde{\mathbf{x}}$ is stable. On the other hand, if at least one of the eigenvalues $\lambda_{n}$ has positive real part, then the solution of Eq. (42) grows with time and the stationary state $\widetilde{\mathbf{x}}$ is unstable. This is the stability criterion.

\section{RESULTS}

The results are stability maps in the plane $\mathrm{Q}_{10} / \mathrm{A}_{\mathrm{p}} \times \theta$ for fixed values of $\mathrm{L}_{\mathrm{p}} / \mathrm{D}$. This maps also display the flooding line given by the flooding correlation given by Eq. (20) with $\mathrm{m}=0.66$ and $\mathrm{C}=0.6$. The range for $\mathrm{Q}_{10} / \mathrm{A}_{\mathrm{p}}$ considered is $0.05 \mathrm{~m} / \mathrm{s}<\mathrm{Q}_{10} / \mathrm{A}_{\mathrm{p}}<0.5 \mathrm{~m} / \mathrm{s}$ and the range for $\theta$ is $\pi / 36 \leq \theta \leq 4 \pi / 9$. The pressure at the pipe entrance is the atmospheric pressure $\mathrm{P}_{\mathrm{atm}}=1.01325 \times 10^{5} \mathrm{~Pa}$ . The working fluids are air and water. The water properties used are $\rho_{1}=998 \mathrm{~kg} / \mathrm{m}^{3}, \mu_{1}=10^{-3}$ Pa.s . The air properties used are $\mu_{\mathrm{g}}=1.8 \times 10^{-5} \mathrm{~Pa} . \mathrm{s}$, $\mathrm{R}_{\mathrm{g}}=287 \mathrm{~J} / \mathrm{kg} . \mathrm{K}$. The air-water interface surface tension used is $\sigma=7.28 \times 10^{-2} \mathrm{~N} / \mathrm{m}$. The pipe diameter $\mathrm{D}=0.052 \mathrm{~m}$.

The stability maps illustrated in Figs. 3, 4 and 5 are for the stationary state with larger void fraction with different $L_{p} / D$ values, where the following symbols represents: +: stable stationary state, *: unstable stationary state and $\mathrm{x}$ : (flooding) no countercurrent flow. The stationary states with the smaller void fraction is always unstable, no matter the value of $\mathrm{Q}_{10} / \mathrm{A}_{\mathrm{p}}, \theta$ and $\mathrm{L}_{\mathrm{p}} / \mathrm{D}$, and its stability maps are not displayed.

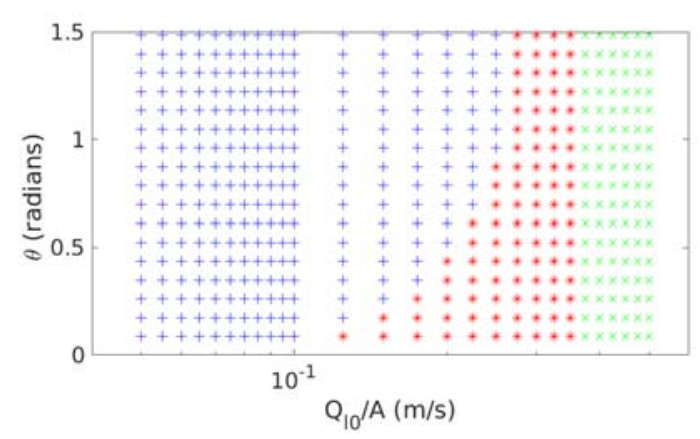

Figure 3. $\mathrm{Lp} / \mathrm{D}=11.5$

\section{DISCUSSION AND CONCLUSIONS}

According to the results, just one stationary state is stable between the two possibilities. The stable stationary state is the one that has the larger void fraction. The flow pattern for this stationary state is always stratified. Figures 3, 4 and 5 reveal that before flooding sets in with the increase of the liquid volumetric flow rate $Q_{l 0}$, the stationary state with large void fraction also looses stability, which is more dramatic for small inclinations and smaller $L_{p} / D$. With inclination increase the unstable region in the plane $Q_{l 0} \times \theta$ decreases significantly, but the increase 
in the parameter $L_{p} / D$ has a minor effect in the decrease of the unstable region.

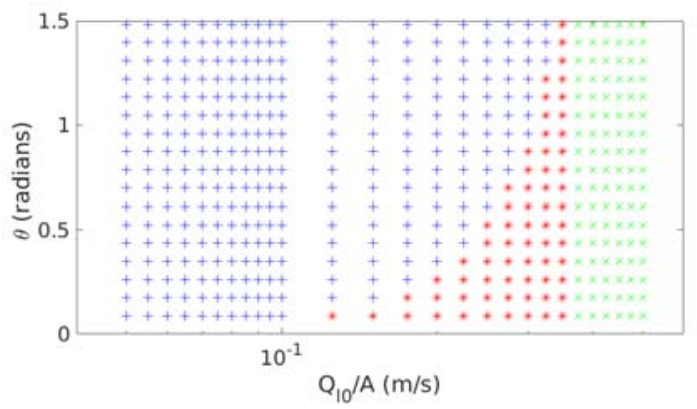

Figure 4. $\mathrm{Lp} / \mathrm{D}=38.5$

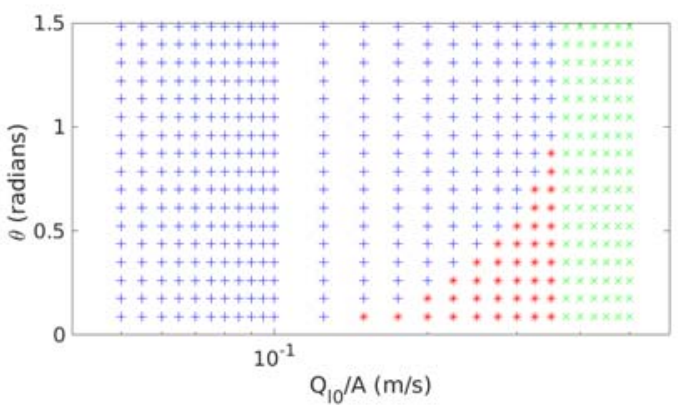

Figure 5. Lp/D $=77$

The results above open the possibility to say that premature shut off results from the absence of PHASE II of the fuel supply process (no stable stationary state to be the steady flow regime in PHASE II). Therefore, the linear stability analysis predicts the region in the parameter space where premature shut off may occur. It may also give an estimate for the time scale for the tank fill up time. Since $j_{l, N}=\tilde{\jmath}_{l, N}+\bar{\jmath}_{l, N}$ and $\bar{J}_{l, N} \propto$ $e^{\lambda_{\max } t}$ where $\lambda_{\max }$ is the finite eigenvalue with the largest real part, Eq. (23) gives an implicit estimate for the period $T_{T}$ to fill up the tank for a given initial fuel depth $\eta\left(t_{0}\right)$. If the real part of $\lambda_{\max }$ is a positive small quantity $\delta \ll 1$, the instability may take a time scale of $O(1 / \delta)$ for its effects to be of order $O(1)$. This time scale may be longer than the tank fill up time scale of order $O(1 / \epsilon)$, and then its effect on the tank fill up process will not be noticed. Based on these arguments, the region in the parameter space where premature shut off effectively occurs is contained in the instability region given by the linear stability analysis and probably smaller.

The counter-current two-phase model for the pipe has a lot of space for improvement. For example, the assumption of uniform pressure at a pipe cross section is physically incorrect when the gravity vector acts in the transverse direction of the flow, since neither the phasic hydrostatic pressure distribution in the transverse direction nor the pressure jump at the gas-liquid interface due to surface tension are taken into account. Another limitation is the assumption of flat gas-liquid interface for the stratified flow, which is incorrect for large pipe inclinations where gravitational effects in the transverse flow direction are weak and surface tension effects dominate, what makes the interface to deform away from the flat configuration. Other points for model improvement are the air-liquid interface friction factor modeling and the use of a more appropriate mean drift velocity correlation for counter-current flow.

\section{REFERENCES}

Banerjee, R., Bai, X., Pugh, D., Isaac, K.M., Klein, D., Edson, J., Breig, W. and Oliver, L., 2002.

"Cfd simulations of critical components in fuel filling systems", SAE Transactions Vol. 111, Section 7: JOURNAL OF PASSENGER CARS: ELECTRONIC AND ELECTRICAL SYSTEMS (2002), pp. 324-340. SAE International.

Banerjee, R., Isaac, K.M., Oliver, L. and Breig, W., 2001. "A numerical study of automotive gas tank filler pipe two phase flow", in: SAE 2001 World Congress, Detroit, Michigan. SAE Technical Paper 2001-01-0732, https://doi.org/10.4271/2001-01-0732.

Chen, N.H., 1979. "An explicit equation for friction factor in pipe". Industrial \& Engineering Chemistry Fundamentals, Vol. 18, No. 3, pp. 296-297.

Chexal, B., Lellouche, G., Horowitz, J. and Healzer, J., 1992. "A void fraction correlation for generalized applications". Progress in nuclear energy, Vol. 27, No. 4, pp. 255-295.

Chexal, B., Merilo, M., Maulbetsch, J., Horowitz, J., Harrison, J., Westacott, J., Peterson, C., Kastner, W. and Schmidt,H., 1997. "Void fraction technology for design and analysis". Technical report, Electric Power Research Institute.,Palo Alto, California.

Deendarlianto, Akiharu Ousaka, A.K. and Fukano, T., 2005. "Investigation of liquid film behavior at the onset of flooding during adiabatic counter-current air-water two-phase flow in inclined pipe". Nuclear Engineering and Design, Vol. 235, pp. 2281-2294.

Fackrell, S., Mastroianni, M. and Rankin, G., 2003. "Model of the filling of an automotive fuel tank". Mathematical and computer modelling, Vol. 38, No. 5-6, pp. 519-532.

Ghiaasiaan, S., Taylor, K., Kamboj, B. and Abdel-Khalik, S., 1995. "Countercurrent two-phase flow regimes and void fraction in vertical and inclined channels". Nuclear science and engineering, Vol. 119, No. 3, pp. 182-194.

Godbille, A., Bayliss, M.T. and Pierson, S., 2007. "A parametric vehicle fuel tank filling system model", in: 2007 SAE World Congress \& Exhibition. SAE Technical Paper 2007-01-1741, 2007, https://doi.org/10.4271/2007-01-1741.

Hibiki, T., 2019. "One-dimensional drift-flux correlations for two-phase flow in medium-size channels". Experimental and Computational Multiphase Flow, Vol. 01, No. 02, pp. 85 - 100. 
Mastroianni, M., Savoni, L., Henshaw, P. and Rankin, G.W., 2011. "Experimental study of fuel tank filling". International Journal of Mechanical and Mechatronics Engineering, Vol. 5, No. 10, pp. 1998 2005.

Ohkawa, K. and Lahey Jr, R., 1980. "The analysis of ccfl using drift-flux models". Nuclear Engineering and Design, Vol. 61, No. 2, pp. 245-255.

Shoham, O., 2005. Mechanistic modeling of gas/liquid two-phase flow in pipes. Society of Petroleum Engineers.

Sinha, N., Thompson, R. and Harrigan, M., 1998. "Computational simulation of fuel shut-off during refueling", in: 1998 International Fall Fuels and Lubricants Meeting and Exposition: San Francisco, California. SAE Technical Paper 981377, https://doi.org/10.4271/981377.

Takeuchi, K., Young, M.Y. and Hochreiter, L.E., 1992. "Generalized drift flux correlation for vertical flow". Nuclear science and engineering, Vol. 112, No. 2, pp. $170-180$. 